\title{
Neurological Disorders from Ambient (Urban) Air Pollution Emphasizing UFPM and $\mathbf{P M}_{2.5}$
}

\author{
Amitava Bandyopadhyay ${ }^{1}$
}

Published online: 10 June 2016

(C) Springer International Publishing AG 2016

\begin{abstract}
Exposure to ambient (urban) air pollution consisting of fine particles is linked with the increased risk of respiratory as well as cardiovascular diseases and cancers. Recent studies have, however, suggested the adverse effects of $\mathrm{PM}_{2.5}$ and especially ultrafine particulate matter (UFPM or $\mathrm{PM}_{0.1}$ : size $<0.1 \mu \mathrm{m}$ or $100 \mathrm{~nm}$ ) in ambient air pollution in the brain and central nervous system (CNS). These particles can travel beyond the pulmonary systems to the CNS and, ultimately, reach the brain that may cause neurological diseases including Alzheimer's disease, Parkinson's disease, and other forms of dementia. The polychlorinated biphenyls (PCBs) can also be linked to neurological disorders identically. An attempt has been made in this article to make an assessment on the roles of UFPM and $\mathrm{PM}_{2.5}$ as well as PCBs on the neurological disorders from exposure to ambient air pollution. Finally, several recommendations are made for future scope of research followed by regulatory enforcement strategies to reduce the occurrence of neurological disorders from exposures of ambient air pollution to humans.
\end{abstract}

Keywords CNS and brain damage $\cdot$ Cognitive decline . Dementia $\cdot$ Neurological disorders $\cdot$ Neurodegeneracy Neurodevelopment

Amitava Bandyopadhyay

amitava.bandy@gmail.com

1 Department of Chemical Engineering, University of Calcutta, 92, A.P.C. Road, Kolkata 700 009, India

\section{Introduction}

Scientists have demonstrated [1-3] that exposure to ambient (urban) air pollution owing to fine particles is linked with the increased risk of various cardiovascular as well as pulmonary disorders. In recent times, studies [4] have shown the negative impacts of ambient air pollution on the brain and central nervous system (CNS) that do not seem to have gained serious attention of the researchers. The polluted air is a complex mixture of several components comprising gaseous compounds and particulate matters. Among these components, particulate matter (PM) of size less than $2.5 \mu \mathrm{m}\left(\mathrm{PM}_{2.5}\right)$ and especially the ultrafine particulate matters (UFPM or $\mathrm{PM}_{0.1}$ ) are mainly responsible for causing neurological diseases to humans including migraine, headache, cerebro-cardiac stroke, Alzheimer's disease (AD), Parkinson's disease (PD), and other forms of dementia. It has also been reported $[5,6]$ that polychlorinated biphenyls (PCBs) including dioxins and furans are linked to dementia and cognitive decline. In the light of the high prevalence of CNS diseases in relation to the increased exposure to outdoor or urban ambient air pollution, this article attempts to make a brief assessment of the neurological disorders reported so far in the literature including neurodegenerative diseases stemming from the ambient air pollution. Finally, several recommendations are put forward for developing action plans for both the developed and developing nations with regard to the future scope of research followed by regulatory enforcement strategies to reduce the increased occurrence of neurological disorders from exposures of ambient air pollution to humans.

\section{Air Pollution: Sources and Definition}

Air pollution is comprised of a diverse mixture of PM (comprising complex mix of solids and liquids including organic 
carbon (OC), elemental carbon (EC), nitrates, sulfates, and metals (e.g., iron, vanadium, nickel, copper, and manganese), gases (e.g., ground level ozone, carbon monoxide (CO), sulfur dioxide $\left(\mathrm{SO}_{2}\right)$, oxides of nitrogen $\left(\mathrm{NO}_{\mathrm{x}}\right)$ ), organic compounds (e.g., methane, non-methane volatile organic compounds (VOCs), polycyclic aromatic hydrocarbons (PAHs), bacterial endotoxins, PCBs including dioxins and furans). Besides outdoor air pollutants are getting introduced into the indoor environment, there are some typical sources of indoor air pollution, for instance, combustion of fuels like wood, coal, oil including kerosene, gas, and products of tobacco. Biological components like cockroach fragments, molds, and mite also contribute to the indoor air pollution. The third important source in this category includes vapors emitting from building materials, household cleaning, and maintenance gadgets as well as furnishings. The PM and ground level ozone among others are emerging as growing risk of urban human health arising from air pollution $[1,4,7-10]$.

The PM is especially relevant for CNS effects and is present in urban air pollution as a mixture of solid particles and liquid droplets suspended in the air. Ambient particles contributing to biological effects are characterized by various sizes ranging from a few nanometers (billionths of a meter) to $10 \mu \mathrm{m}$ (millionths of a meter) in diameter: $\mathrm{PM}_{2.5-10}$ (coarse particles with diameter of 2.5 to $10 \mu \mathrm{m}$ ), $\mathrm{PM}_{2.5}$ (fine particles less than $2.5 \mu \mathrm{m}$ ), and UFPM (ultrafine particulate matters; $<0.1 \mu \mathrm{m}$ or $100 \mathrm{~nm}$ or $\mathrm{PM}_{0.1}$ ). The respirable fraction of the $\mathrm{PM}$ is $\mathrm{PM}_{2.5-10}$. The $\mathrm{PM}_{2.5-10}$ originates from many sources such as paved and unpaved road dusts, dusts from construction and demolition activities, emissions due to wear of tires, wood combustion, agricultural dust, and mining operations. In an urban city, however, the agricultural dust and dusts from mining operations would not be present. On the other hand, the $\mathrm{PM}_{2.5}$ is produced from gas and condensed vapors that originate from various combustion sources and industrial activities. As a result, these particles comprise both organic and inorganic compounds, such as ammonium compounds, carbon, sulfates, nitrates, lipo-polysaccharides, metals, and water. Major sources of $\mathrm{PM}_{2.5}$ are emissions from automobiles, fuel combustion during cooking at home as well as at roadside eateries, open burning of garbage (municipal solid waste, MSW), industrial activities (petroleum oil refineries, metal processing facilities, power plants), and wildfires $[1,4,7-10]$.

The UFPMs or nano-sized particles (NPs) of less than $0.1 \mu \mathrm{m}$ are mostly combustion-derived NPs, which can be produced by internal combustion engines, power plants, incinerators, and thermo-degradation of other sources (such as open burning of garbage or MSW consisting of all kinds of plastics, rubbers, and other polymers). They can carry soluble organic compounds, PAHs, PCBs including dioxins, furans, and oxidized transition metals on their surfaces [1, 4, 7-10]. Thus, UFPM, therefore, assumes considerable importance in studies of neurological disorders from air pollution both in the form of particulate phase as well as gaseous (molecular) phase.

Interestingly, an estimate indicates that the air pollution ranks among the top ten leading risk factors for mortality that is incidentally responsible for deaths of about 3 million people each year [4]. The global air pollution is contributed from traffic-related urban air pollution in which the diesel exhaust (DE) constitutes the important component [11]. It comprises more than 40 air toxics including UFPM, and its exposure is often used for measuring the traffic-related air pollution. Greenbaum [12] reported that the contribution from the automobile emissions for most of the air pollutants in the ambient levels was ranging from 25 to $40 \%$. Furthermore, contributions of the pollutants in a few cases, such as CO, UFPM, and 1,3-butadiene from automobiles were considerably higher. In another study, the emissions of PM from automobiles in Asian countries were estimated to be about 25 to $35 \%$ [13]. The rest of the emissions also play an important role in maintaining the ambient air quality (AAQ) of an urban city. In Kolkata, open burning of agricultural waste, biomass, firewood, and discarded automobile tires and tubes could be noticed for the purpose of heating during winter in slum areas where economically constrained people live in. In addition, open burning of garbage or MSW (containing all kinds of plastic) as mentioned earlier could be seen any time in the year (especially in and around railway tracks, railway stations in Kolkata, India) as a measure of managing this growing waste stream. Emissions from open burning of these sources mainly emit UFPM, $\mathrm{PM}_{2.5}, \mathrm{CO}, \mathrm{SO}_{2}, \mathrm{NO}_{\mathrm{x}}$, PAHs, and PCBs including dioxin and furans. The research data are scanty on these emissions as explained next. Estimates of primary emissions of $\mathrm{PM}_{2.5}$ in Indian urban cities from combustion of fossil fuels (coal, diesel, and gasoline) were shown to be 25 to $33 \%$ in Delhi and 37 to $57 \%$ in Kolkata, while emissions of $\mathrm{PM}_{2.5}$ from combustion of biomass were shown to be 7 to $20 \%$ in Delhi and 13 to $18 \%$ in Kolkata from chemical mass balance (CMB) using monitored data generated during March 2001 to January 2002 [14]. It was also reported [14] that the emissions from biomass and coal combustions were relatively higher during winter due to higher heating requirements and poor dispersion for shorter inversion heights. It was further concluded therein that besides contribution from mobile sources, the biomass combustion contributed significantly and in many instances in equal proportions, but road dust dominated at times. In a study carried out by the Asian Development Bank under the aegis of West Bengal Pollution Control Board reported [15] that the emissions of $\mathrm{PM}_{2.5}$ in Kolkata Metropolitan Area (KMA) was about 21.2 \% from the combustion of diesel and petrol-driven vehicles and about $10.1 \%$ from open burning of biomass (trash) based on CMB source apportionment studies. This report also mentioned that only $5 \%$ of the total MSW was composted while $94 \%$ was dumped in open areas and $25 \%$ (ca.) of which was burned, 
on average, in India including KMA. Significant emission of $\mathrm{PM}_{2.5}$ from domestic cooking in slum areas were also reported therein. Further, emissions of $\mathrm{PM}_{2.5}$ from slum cooking and biomass burning were reported to be 1211 and 4388 TPY, respectively, during 2003 which were projected to be around 2300 and 8330 TPY in 2014 with a yearly increment of $5.5 \%$ without any control measures. The health effects of urban air pollution in Kolkata were only studied in the lungs alone with indications of inflammatory and allergic lung response and microscopic hemorrhage of lung [15]. The report recommended banning of open burning of biomass. Since then, appropriate measures to control the open burning of biomass and others as well as health-related studies from urban air pollution for improving the AAQ does not seem to have been undertaken in Kolkata.

\section{Assessing Neurological Disorders from Ambient Air Pollution due to UFPM and $\mathbf{P M}_{2.5}$}

Block and Calderón-Garcidueñas [7] reported that air pollution was a prevalent pro-inflammatory stimulus to the CNS which was overlooked so far as a risk factor for neurodegenerative diseases. It was estimated that 29 million people were exposed to $\mathrm{PM}_{10}$ and 88 million were exposed to $\mathrm{PM}_{2.5}$ in the USA alone. The levels of UFPM were unmonitored and unregulated in the USA; exposure was, however, estimated to be high.

Scientists have demonstrated [1] that exposure to ambient (urban) air pollution owing to fine particles is linked with the increased risk of pulmonary and cardiovascular disorders to a wide variety such as reduced oxygen supply to the heart (myocardial ischemia), heart attack, heart failure, stroke, arrhythmia and sudden death, other cardiovascular diseases, venous thrombosis (blood clots), respiratory illnesses [16, 17], and lung cancers [9]. Recent studies [4] have, however, suggested the negative impacts of ambient air pollution in the brain and CNS that do not seem to have gained serious attention of the researchers. The UFPM and $\mathrm{PM}_{2.5}$ present in the polluted air are inhaled on a regular basis due to air pollution and are of major concern, as these particles can travel beyond the pulmonary systems, crossing the blood-air barrier (BAB) of the lungs to the CNS and, ultimately, reach the brain crossing the blood brain barrier (BBB) [7].

In an investigation carried out by Levesque et al. [18] using Male Fischer 344 rats exposed to subchronic DE by inhalation over 6 months reported to cause neuroinflammation and elevated early markers of neurodegenerative disease. A study carried out on 2715 children aged from 7 to 10 years from 39 schools in Barcelona, Spain, exposed to high and low traffic-related air pollution indicated smaller improvement in cognitive development [19]. In another study carried out on 50 Mexico City Metropolitan Area (MCMA) children having ages $13.4 \pm 4.8$ years on urban air pollution reveled decreased attention, short-term memory, and below-average levels of intelligent quotient [20]. A different study performed in the same area (MCMA), 139 children having ages $11.91 \pm 4.2$ year were reported [21] to show an early brain imbalance in oxidative stress, inflammation, innate and adaptive immune response-associated genes, and BBB breakdown from exposures of urban air pollution. Air pollution was shown [22] to adversely affect the cognitive- function and- performance in elderly women, in which the impact of the traffic exposure was significant to those carrying the apolipoprotein $\mathrm{E}$ (APOE) $\varepsilon 4$ allele the most prevalent genetic risk for AD. The exposure to $\mathrm{PM}_{2.5}$ from ambient air pollution on 1403 community-dwelling older women without dementia showed reduction of brain volumes especially the white matter [23].

Particle size, surface characteristics, and deposition site are the three important factors governing the deposition behavior, clearance mechanisms, cell entry, and translocation to extrapulmonary sites of particles. Brownian motion or diffusion further determines the deposition site of particles of size up to $20 \mathrm{~nm}$. Relatively larger particles are deposited by interception and impaction mechanism as opposed to Brownian diffusion. Therefore, these nano-particles are behaving like molecules depositing in the upper respiratory tract directed towards the olfactory mucosa causing biological and toxicological effects. The surface property affecting the kinetics is another important property of the NPs. Extensive particle characterization can only explain the physicochemical behaviors of the site of deposition leading to the deterioration of the cell and associated factors [10].

Block and Calderón-Garcidueñas [7] identified multiple potential pathways for damage to the brain and central nervous system using animal and human models including neuroinflammation, altered immune system responses, aggregation of proteins, and the direct toxic effects of the pollutants themselves. They theorized that the inflammation in the brain and changes in immune system cells, such as microglia, caused by the increased pro-inflammatory signals in the whole body due to exposure to air pollution. This ultimately leads to brain - or BBB - damage that eases entry of air pollutants followed by accumulation in the brain in tandem, in addition to the toxicity that these pollutants have on brain tissues. The exact mechanistic pathways are yet unexplored [7].

Many routes have so far been theorized [10] for transport of $\mathrm{PM}_{2.5}$ and especially UFPM to the CNS and brain causing neurological disorders leading to relevant diseases, but the exact transport mechanism is yet to be explored. It was further reported that metal (such as nickel and vanadium) accumulation and tissue damage occurred in a gradient fashion (olfactory mucosa $>$ olfactory bulb $>$ frontal cortex). It indicated that the nasal pathway was a portal of entry. It is noteworthy that the olfactory dysfunction is a common feature in neurodegenerative diseases indicating exposure coupled with inhalation 
of air pollutants may be linked etiologically to olfactory neuropathology.

Investigations undertaken, are sparse, to assess the role of $\mathrm{PM}_{2.5}$ and UFPM in neurological diseases stemming from air pollution on humans including migraine, headache, cerebrocardiac stroke, $\mathrm{AD}, \mathrm{PD}$, and other forms of dementia. The two intrinsic effects of ambient air pollution such as the oxidative stress and neuroinflammation in both humans and animals are confirmed by in vitro studies. Age has been considered as one of the most relevant factors adversely influencing the neurotoxic outcomes [10].

These studies reported to suggest [4] that air pollution may cause developmental neurotoxicity leading to neurodevelopmental disorders, including autistic disorders and neurodegenerative diseases. The preclinical and clinical evidences showed that air pollution, especially traffic-derived pollution, was causing CNS damage. Both, the physicochemical characteristics of the particle and toxic compounds adsorbed on it might be responsible for the damage. The time of exposure also has an important role to play on the damage. For an acute exposure, the minimum dose of the pollutant could be handled, but the same dose chronically administered could lead to an oxidative stress producing the neurodegeneration. The effect of the pollutants on the CNS can be chronic which can begin in early childhood and may accumulate over age [1].

\section{PCBs and POPs in Ambient Air and Neurological Disorders}

Diesel exhaust and automobile emissions have so far been given serious attention for causing adverse health effects including neurodevelopment and neurodegenerative disorders. But emissions from open burning of garbage containing plastics and similar other components in developing nations as mentioned earlier is contributing equally to the ambient air pollution and health damages. Since, dioxins and furans introduced into the ambient air are especially generated from the open burning of all kinds of plastics and rubbers, neurological disorders stemming from these molecules (or broadly from PCBs) are reviewed [24] here.

The PCBs are industrial chemicals used for many years in a legion of applications, such as lubricants, insulators, electrical equipment, and paint additives. The PCBs are also known as persistent organic pollutants (POPs) since they are not easily broken down and hence continue to contaminate the general environment persistently. These were found to cause cancer. Furthermore, PCBs were found to interfere with the normal brain development and thyroid hormone function. Two PCBs, as man-made combustion products, such as dioxins and furans are important from the perspective of ambient air pollution and neurodegeneracy. Open burning of garbage and MSWs containing plenty of plastics of any form, waste tires/tubes, other polymers in developing nations as in Kolkata, India, can significantly generate PCBs in the form of dioxins and furans in the ambient air. The toxicity of PCBs on CNS and brain, therefore, assumes considerable importance.

There are epidemiological studies [25] on the toxicity of PCBs on developing brain, but studies demonstrating the association of PCB including furans exposure of adult with dementia/cognitive impairment are limited. Three such different studies reported on PCB exposure were (i) from cooking oil contaminated with PCBs and polychlorinated dibenzofurans (PCDFs or furans in short) leading to reductions in attention and memory functions among women [6], (ii) fish consumption as an environmental exposure leading to memory and learning impairments compared to controls [26], and (iii) a group of occupationally exposed workers showing enhanced dementia [27]. Several epidemiological studies [28-32] have so far linked the exposure to PCBs and other POPs to inflammation, diabetes, and metabolic syndrome. Atherosclerosis and obesity are also linked with low-dose of these chemicals. Given that these diseases are themselves risk factors for dementia/cognitive impairment, these chemicals thus effect on the dementia/cognitive impairment mediated in part through these risk factors, as well as through direct PCB effects on the brain. The exact mechanisms for causing neurodegeneration by PCBs are not yet explored; however, it is reported [33-35] that PCBs can lead to oxidative stress as well as free radical production and are linked to inflammation of endothelial cells, as well as impairing the synthesis of longchain fatty acids. On the other hand, neurological diseases such as dementia and cognitive decline are reported due to the reduced level of long-chain fatty acids from the body threshold level. Therefore, PCBs are linked to dementia and cognitive decline via impairment of synthesis of long-chain fatty acids. Furthermore, the functioning of the thyroid hormone is reported to be adversely affected by the PCBs that may lead to cognitive impairment.

\section{Assessing the Ambient Air Pollution Exposure}

In assessing the exposure to ambient air pollution, it is reported [9] that the outdoor or urban AAQ monitoring is routinely conducted worldwide in large human populations for the "criteria" air pollutants comprising the gases $\mathrm{SO}_{2}, \mathrm{NO}_{\mathrm{x}}, \mathrm{CO}$, ozone, and particulate matters such as lead and $\mathrm{PM}_{10}$ with the recent inclusion of $\mathrm{PM}_{2.5}$. These air pollutants generally originate almost from the same source like combustion facilities and even interact with each other chemically. As the concentrations of these pollutants in the ambient air are correlated in space and time, it is difficult to isolate the biological effects of individual pollutants from the studies of their exposure assessment that solely relies on environmental monitoring network 
data. Monitoring networks installed in urban areas for spatially homogenous pollutants (e.g., $\mathrm{PM}_{2.5}$ ) allow estimation of community-wise average exposures for recording data consistently as a function of time but these networks are seldom designed with the adequate number of monitoring stations for spatially heterogeneous pollutants. This has resulted in the generation of monitored data for the pollutants avoiding the proximity to sources that determines the peak exposures. For instance, UFPM, black smoke (a marker of exhaust particles) and very importantly dioxins, furans (PCBs/POPs) (markers of open burning of garbage containing all kinds of plastics/rubbers and other polymeric materials) are not at all monitored as these are not included in the AAQ standards, and $\mathrm{CO}$ and $\mathrm{NO}_{\mathrm{x}}$ (markers of vehicle exhaust) are not monitored properly simulating the real situations. These inadequacies are further augmented by the lack of source apportionment studies that could inform possible sources of pollutant exposures in any urban settings.

\section{Knowledge Gaps and Future Scope of Research}

Critical appraisal of the current literature indicates that the scientists only start from their fields on studies of environmental impacts on public health. Further, investigations often carried out by the atmospheric scientists on public health show the statistical results only ignoring the pathological analyses. In contrast, medical experts seldom analyze the air quality data in the manner it is desired to draw proper and holistic conclusions. However, the scopes of research on the emerging risks of neurodevelopment and neurodegenerative diseases (e.g., dementia and cognitive impairment) stemming from the ambient (outdoor/urban) air pollution are demonstrated elsewhere in the literatures $[1,9,10,36]$. In order to fill these knowledge gaps in the current literature, an attempt has been made to put forward recommendations for the future scope of research reviewing those listed in the literatures $[1,9,10,36]$ as well as introducing newer concepts and ideas given in the following sub-sections. Similar recommendations could also be put forward for indoor air exposures especially due to kitchen exhausts, since these emissions likely to affect similar to the neurodegeneracy and other health disorders of women engaged in the kitchen in the developing nations after suitably taking into consideration of the indoor air quality parameters and monitoring protocols.

1. Designing AAQ monitoring network, monitoring of AAQ parameters, characterization of $P M$, and source apportionment studies

(a) AAQ monitoring network design [9]

Monitoring network should be designed keeping the spatially homogenous as well as heterogeneous pollutants in mind. The exposure of the pollutants to the living receptors should be covered adequately in the network design. Monitoring of AAQ parameters should be carried out rigorously at well-defined monitoring stations identified in the monitoring network.

(b) Monitoring of AAQ parameters [36]

Besides monitoring of the regular traditional AAQ parameters, parameters that are assumed to be important in causing dementia and cognitive decline should also be monitored regularly. These are detailed as follows.

(i) Monitoring of UFPM in addition to $P M_{2.5-10}$ and $\mathrm{PM}_{2.5}$ [36]

Regular monitoring of UFPM is recommended in addition to $\mathrm{PM}_{10}$ and $\mathrm{PM}_{2.5}$.

(ii) Determination of heavy metal content and anions in airborne PM [36]

Airborne particles may contain heavy metals and anions, which can pose a risk to human health and the environment. Thus, heavy metal content should be estimated in the UFPM, $\mathrm{PM}_{2.5}$, and $\mathrm{PM}_{2.5-10}$ samples collected during monitoring.

(iii) Determination of $\mathrm{pH}$ and dissolution property of the PM collected

The $\mathrm{pH}$ of collected particulates (UFPM, $\mathrm{PM}_{2.5}$, and $\mathrm{PM}_{2.5-10}$ ) should be measured using a water wash from the filter media. Determining the variation of $\mathrm{pH}$ during various seasons at various monitoring stations could be revealing [36]. The dissolution properties of PM collected are proposed here to be thoroughly investigated using distilled water and various other organic solvents (polar and non-polar) to simulate conditions in vivo of humans

(iv) Determination of PCBS/POPs (dioxins and furans) and PAHs/VOCs in the ambient air

Determination of PCBs/POPs (including dioxins and furans) and PAHs/VOCs during various seasons at various monitoring stations could lead to interesting conclusions in the urban areas. Monitoring of these components would be seemingly important at strategic locations emphasized during winter in developing nations as mentioned earlier.

(c) Characterization of UFPM, $P M_{2.5}$, and $P M_{2.5-10}$ [36]

After monitoring of UFPM, $\mathrm{PM}_{2.5}$, and $\mathrm{PM}_{2.5-10}$, the collected particles should be characterized by various analytical methods importantly but not limited to Fourier transform infrared (FTIR) spectroscopy for determining the functional groups, field emission scanning electron microscopy (FE-SEM) and 
scanning electron microscopy (SEM) equipped with an energy dispersive X-ray (EDX) for analyzing the topographic microstructure and elemental mapping in a cross-section, inductively coupled plasma mass spectrometry (ICP-MS) for detecting metals and several non-metals at concentrations as low as one part in $10^{15}$ (part per quadrillion, ppq), X-ray fluorescence (XRF) for elemental analysis and chemical analysis, X-ray diffraction (XRD) for identification of crystalline materials, and Brauner-Emmette-Teller (BET) surface area analysis for determining the surface area and pore volume. Attempts may also be taken to determine the size distribution of the particles collected. In addition, these particles should be analyzed for black carbon (BC), EC, and OC.

(d) Thermal impact on the pollutants and health effect

The thermal behavior nowadays would play an important role in the urban ambient air. This issue has never been given any serious attention by the researchers, regulators, and planners, and hence, the thermal impact on the pollutants followed by their health effects (dementia and cognitive decline) has been included first time in AAQ studies here. In urban society, the numbers of automobiles are increasing at an alarming rate. Many of the vehicles are fitted with the air-conditioning systems that are used to get comfort from outdoor heat. In addition, increased numbers of multistoried residential buildings and shopping malls are being constructed fitted with the large air-conditioning systems for getting comfort from heat. Incidentally, these two are increasing the discharge of hot exhaust and hot air in to the urban ambient air day-by-day especially during summer. For example, the urban cities of developing nations (such as Kolkata, India) are facing this challenge. Now, therefore, the health effects of PMs especially the UFPM and $\mathrm{PM}_{2.5}$ under hot condition need be investigated. The physicochemical behaviors of these particles have so far been given attention. Now the effects of temperature and heat on the pollutants (for instance, UFPM and $\mathrm{PM}_{2.5}$ ) demand serious attention in the loop of the studies of AAQ upon dementia and cognitive impairment among other health effects.

(e) Source apportionment studies

Monitoring should follow source apportionment studies extensively covering the parameters specified here in this article in addition to the traditional AAQ parameters to identify the contributing sources prior to taking any ad hoc decision.
2. Identifying the presence of neurotoxic components in ambient air

(a) To assess the physicochemical properties and thermal effects of the additional AAQ parameters (specified in serial number $1 \mathrm{~b}$ earlier) for causing neuroinflammation or neurotoxic effects in the brain and CNS.

(b) To perform investigations on the toxico-kinetics of UFPM and PM2.5 on neuropathology to examine the transport mechanism of pollutants into the portal system to the brain followed by the biotransformation, distribution, and elimination of pollutants from the brain, and finally the influences of particle size and composition on transport and elimination from the brain [9].

(c) To identify the populations (aged, young, genotype, socio-economically constrained groups, high stress, and CNS disease) that are vulnerable to CNS and brain damage due to air pollution through properly designed epidemiological studies [9].

3. Investigating into the cellular and molecular mechanisms of neuropathology [9]

(a) To investigate into the role of peripheral inflammatory and neurohormonal mediators (from respiratory tract and/or blood) on the effects of air pollution on neuroinflammation, CNS pathology, bio-kinetics, and behavior.

(b) To explore the role of peripheral immune cells in the air pollution-affected areas of CNS and brain.

(c) To develop mechanisms affecting the cells of CNS (astrocyte, microglia, oligodendrocyte, and neurons) induced by the air pollution and their role in pathology.

(d) To explore the effect of air pollution on BBB and $\mathrm{BAB}$ of the lungs and the consequent impacts on CNS and the brain.

(e) To determine the interactions between CNS and other diseases induced by the air pollution.

\section{Epidemiological research on humans [9]}

(a) To explore the effects of specific air pollutants on increased risk for neurodevelopmental disorders (e.g., autism), neurodegenerative diseases (e.g., $\mathrm{AD}, \mathrm{PD}$, and other forms of dementia), and mental disorders (e.g., depression) in humans.

(b) To ascertain as to whether the observed effects of air pollution on the CNS occur downstream or independent of cardiovascular effects or cerebrovascular damage in adult cohorts that are well-characterized.

(c) To utilize estimates of refined exposure for examining the long-term effects of air pollution on the brain 
damage in various age groups developing spatiotemporal models for various air pollutants.

(d) To investigate into the effects of acute exposure of air pollutants on CNS, such as during reported peak hours of ozone or PM using multiple temporal points.

(e) To examine the effects of air pollution on the brain in sensitive subgroups, including genetically susceptible populations, to explore the plausible mechanisms.

(f) To determine the sub-clinical (neurological and neurobehavioral) outcomes of importance in influencing the CNS taking the advantage of magnetic resonance imaging (MRI) and other neuroimaging technologies that can provide information regarding mechanisms.

\section{Developing experimental program}

(a) To develop and use appropriate standardized protocols and good quantitative descriptions of real-world exposure conditions since testing air pollutants toxicity in humans (dosimetry) are lacking. It is further suggested to develop novel methods for detecting exposure and dosimetry calculation $[1,9]$.

(b) To develop experimental programs realistically taking into consideration of the pollutants present in the ambient air in terms of composition and concentration covering place- and source-specific emissions. For instance, the neurological disorders need be investigated for automobile emissions as well as emissions generated from open burning of garbage and others as detailed earlier.

(c) To undergo adequate exposure assessment, and to identify the separate roles of insoluble versus soluble components in mediating responses to CNS and brain [9].

(d) To assess the interaction between the gene and the environment for exploring the possible mechanisms and the molecular pathways connecting ambient air pollution with CNS damage because genetic susceptibility has shown to respond to air pollution $[1,9]$.

(e) To investigate into the roles of gene-air pollution interactions followed by gene-by-gene interactions and epigenetic mechanisms [1].

(f) To incorporate the CNS health outcomes, such as dementia ascertainment and cognitive performance, into the existing human/epidemiology exposure assessment campaigns [9].

(g) To support near-lifetime exposure studies in animals (mammal) [9].

(h) To include studies of behavioral endpoints, an important area of interest in human and animal studies [9].

(i) To undertake research projects with cross-national collaborations including the developing nations for developing standardized data bank on neurological disorders stemming from urban ambient air pollution especially due to UFPM and $\mathrm{PM}_{2.5}$ including PCBs/ $\mathrm{PAHs}$ clearly identifying the monitoring stations for the two different sources such as automobiles and open burning of garbage as indicated earlier for drawing cogent conclusions. Uses of fireworks in festive seasons play an important role in short-term deterioration of AAQ in many urban cities of India. It is, therefore, proposed here to include studies on the impact of fireworks on AAQ and dementia and cognitive decline.

(j) To formulate action plans for commencing the crossnational borderline areas of research involving neuroscience, medical science, microbiology, pharmaceutical technology, ambient air pollution, air pollution control, chemical and bio-chemical engineering, and statistics.

6. Collection and analysis of samples for airborne microorganisms

Critical appraisal of the existing literature revealed that the AAQ is strongly influenced by the presence of airborne microorganisms or bioaerosols [37]. Sampling airborne microorganisms should be conducted according to the methods described in the literature [37]. In addition, newer concepts could be introduced in the analytical program. For instance, the morphologies of the bioaerosols attached to PM, such as UFPM, $\mathrm{PM}_{2.5}$, and $\mathrm{PM}_{2.5-10}$, should be rigorously investigated. After obtaining these results, the relationships among airborne microorganism counts and PM, temperature, rainfall, and human settlements (capacity and quality) should be estimated. Sampling and analysis of airborne microorganisms and bioaerosols could help assessing the overall impacts of PM and other chemicals as well as bioaerosols on CNS and brain damage. The profile of the bioaerosols could be developed, if the station-wise monitoring data are superimposed on the local land use map of the urban city. This issue may be very important especially at strategic locations (slums) where people of lower income group reside in the developing nations and similar other places.

\section{Regulatory enforcement strategies}

(a) To develop enforcement strategies for improving the ambient air quality standards, minimizing personal exposures, and redesigning of engine and fuel technologies that would reduce the level of air pollution and its consequences for neurological morbidity and mortality [1].

(b) To develop health policies derived from chemical and toxicological characteristics of PM on neurodevelopment and neurodegenerative disorders (cognitive impairment/dementia).

(c) To formulate neurological health registry of a specific urban city based on its AAQ. This is to be 
conceived through the registrations of patients on the neurological disorders made to the medical practitioners (at individual, and hospital/nursing home levels) coupled with the AAQ in which the patient is living. Besides gathering other health data of such patients, this registry will help in identifying the impact AAQ upon CNS and brain damage.

(d) To review the regulatory measures already taken to implement cleaner fuel (such as CNG) technologies for automobiles as emissions from such engines would generate UFPM to a larger extent coupled with PAHs and the toxicity may be more than using other fuels so far as the neurodevelopment and neurodegenerative disorders are concerned. Nations those have undertaken such fuel change options may be suffering from increased prevalence of neurodevelopment and neurodegenerative disorders compared to uncontrolled situations. Initiatives in performing research investigation may be undertaken by the respective nations under a mutually convenient strategy keeping in view the socio-economic impacts in the change of fuel.

(e) To take proactive action by the respective governments in establishing the Urban Environmental Management (UEM) department or center in academic institutions located at urban cities in developing nations as in India where such academic department is missing, though the government is composed of Ministry of Urban Affairs both at central and state level. It is noteworthy to mention about the issuance of a notice at the central levels by the University Grants Commission of India in December 11, 2015 for including a module on ill-effects of fireworks in the paper Environmental Studies and other associated programs [38]. As a part of similar other urban issues like solid waste management, drinking water quality management, sewage management, urban carrying capacity, and urban stress management shall be designed under UEM course and curriculum that would help the urban ministries at national as well as state levels in providing indigenize policy recommendations for proper urban management. The model may be applied to any nations lacking such decision support system. This department or center further has to undertake inter-disciplinary academic programs at post graduate levels followed by extensive research programs collaborating with institutes undertaking researches in neurosciences and AAQ both nationally and internationally.

(f) To undertake awareness program by the regulators and UEM academic departments to make people aware on the health consequences of air pollution generated from various sources keeping in mind that the perception of the public in weaker economies is in general in its nascence in the developing nations.

\section{Conclusions}

Recent studies suggested the adverse effects of $\mathrm{PM}_{2.5}$ and especially UFPM in the ambient air pollution on the brain and central nervous system (CNS) besides respiratory/ cardiovascular diseases and lung cancers. These particles can cause neurological diseases including Alzheimer's disease, Parkinson's disease, and other forms of dementia. The PCBs can also be linked to similar neurological disorders. An assessment was made in this article on the role of UFPM and $\mathrm{PM}_{2.5}$ as well as PCBs on the neurological disorders from exposure to ambient air pollution. Finally, several recommendations were made for future scope of research followed by regulatory enforcement strategies to reduce the occurrence of neurological disorders from exposures of ambient air pollution to humans.

\section{References}

1. Genc S, Zadeoglulari Z, Fuss SH, Genc K. The adverse effects of air pollution on the nervous system. J Toxicol. 2012, Article ID 782462,23 pages.

2. Calderón-Garcidueñas L, Calderón-Garcidueñas A, Torres-Jardón R, Avila-Ramírez J, Kulesza RJ, Angiulli AD. Air pollution and your brain: what do you need to know right now. Primary Health Care Res \& Dev. 2015;16(04):329-45.

3. Bos I, Boever PD, Panis LI, Meeusen R. Physical activity, air pollution and the brain. Sports Med. 2014;44(11):1505-18.

4. Costa LG, Cole TB, Coburn J, Chang Y-C, Dao K, Roque P. Neurotoxicants are in the air: convergence of human, animal, and in vitro studies on the effects of air pollution on the brain. BioMed Res Int. 2014, Article ID 736385, 8 pages.

5. Schantz S, Widholm J, Rice D. Effects of PCB exposure on neuropsychological function in children. Environ Health Perspect. 2003;111(3):357-576.

6. Lin K, Guo N, Tsai P, Yang C, Guo YLL. Neurocognitive changes among elderly exposed to PCBs/PCDFs in Taiwan. Environ Health Perspect. 2008;116:184-9.

7. Block ML, Calderón-Garcidueñas L. Air pollution: mechanisms of neuroinflammation \& CNS disease. Trends Neurosci. 2009;32(9): 506-16.

8. Moulton PV, Yang W. Air pollution, oxidative stress, and Alzheimer's disease. J Environ Publ Health. 2012, Article ID 472751, 9 pages.

9. Block ML, Elder A, Auten RL, Bilbo SD, Chen H, Chen J-C, et al. The outdoor air pollution and brain health workshop. Neurotoxicology. 2012;33(5):972-84.

10. Loane C, Pilinis C, Lekkas TD, Politis M. Ambient particulate matter and its potential neurological consequences. Rev Neurosci. 2013;24(3):323-35.

11. Ghio AJ, Smith CB, Madden MC. Diesel exhaust particles and airway inflammation. Curr Opin Pulm Med. 2012;18(2):144-50. 
12. Greenbaum DS. Chapter 5: Sources of air pollution: gasoline and diesel engines, in air pollution and cancer; Eds. Straif K, Cohen K, Samet J. IARC Scientific Publications: 161. International Agency for Research on Cancer. 2013.

13. HEI. Outdoor air pollution and health in the developing countries of Asia: a comprehensive review. Special Report 18. Boston, MA: Health Effects Institute. 2010.

14. Chowdhury Z, Zheng M, Schauer JJ et al. Speciation of ambient fine organic carbon particles and source apportionment of $\mathrm{PM}_{2.5}$ in Indian cities. J Geophys Res: Atmosph. 112(D15), D15303. doi:10. 1029/2007JD008386. 2007.

15. Strengthening Environmental Management at the State Level (cluster); Component E - strengthening environmental management at West Bengal Pollution Control Board; Final Report, Volume V: Air Quality Management, Asian Development Bank, TA No. 3423IND, prepared by Intercontinental Consultants and Technocrats Pvt. Ltd., India in association with Ballofet International LLC, USA and Water \& Power Consultancy Services (India) Ltd. November 2005.

16. Brook RD. Air Pollution. What is bad for the arteries might be bad for the veins. Arch Intern Med. 2008;168(9):909-11.

17. Baccarelli A, Martinelli I, Zanobetti A, Grillo P, Hou L, Bertazzi $\mathrm{PA}$, et al. Exposure to particulate air pollution and risk of deep vein thrombosis. Arch Intern Med. 2008;168(9):920-7.

18. Levesque S, Surace MJ, McDonald J, Block ML. Air pollution \& the brain: subchronic diesel exhaust exposure causes neuroinflammation and elevates early markers of neurodegenerative disease. $\mathrm{J}$ Neuroinflammation. 2011;105:1-10.

19. Sunyer J, Esnaola M, Alvarez-Pedrerol M, Forns J, Rivas I, LópezVicente M, et al. Association between traffic-related air pollution in schools and cognitive development in primary school children: a prospective cohort study. PLoS Med. 2015;12(3):e1001792.

20. Calderón-Garcidueñas L, Mora-Tiscareño A, Franco-Lira M, Zhu $\mathrm{H}, \mathrm{Lu} \mathrm{Z}$, Solorio E, et al. Decreases in short term memory, IQ, and altered brain metabolic ratios in urban Apolipoprotein $\varepsilon 4$ children exposed to air pollution. J Alzheimers Dis. 2015;45(3):757-70.

21. Calderón-Garcidueñas L, Vojdani A, Blaurock-Busch E, Busch Y, Friedle A, Franco-Lira M, et al. Air pollution and children: neural and tight junction antibodies and combustion metals, the role of barrier breakdown and brain immunity in neurodegeneration. $\mathrm{J}$ Alzheimers Dis. 2015;43(3):1039-58.

22. Schikowski T, Vossoughi M, Vierkötter A, Schulte T, Teichert T, Sugiri $\mathrm{D}$, et al. Association of air pollution with cognitive functions and its modification by APOE gene variants in elderly women. Environ Res. 2015;142:10-6.

23. Chen JC, Wang X, Wellenius GA, Serre ML, Driscoll I, Casanova $\mathrm{R}$, et al. Ambient air pollution and neurotoxicity on brain structure: evidence from women's health initiative memory study. Ann Neurol. 2015;78(3):466-76.

24. Stein J, Schettler T, Rohrer B, Valenti M. Ed. Myers N. environmental threats to healthy aging: with a closer look at Alzheimer's \& Parkinson's diseases. Greater Boston Physicians for Social Responsibility and Science and Environmental Health Network. Chapter 7: Environmental factors in the development of dementia: focus on Alzheimer's disease and cognitive decline. 2008.
25. Liu J, Lewis G. Environmental toxicity and poor cognitive outcomes in children and adults. J Environ Health. 2014;76(6):130-8.

26. Schantz SL, Gasior DM, Polverejan E, McCaffrey RJ, Sweeney AM, Humphrey HE, et al. Impairments of memory and learning in older adults exposed to polychlorinated biphenyls via consumption of Great Lakes fish. Environ Health Perspect. 2001;108:60511.

27. Steenland K, Hein MJ, Cassinelli RT, Prince MM, Nilsen NB, Whelan AZ, et al. Polychlorinated biphenyls and neurodegenerative disease mortality in an occupational cohert. Epidemiology. 2006;17(1):8-13.

28. Lee DH, Lee IK, Song K. A strong dose-response relation between serum concentrations of persistent organic pollutants and diabetes: results from the National and Examination Survey 1999-2002. Diabetes Care. 2006;29(7):1638-44.

29. Lee DH, Lee IK, Jin SH, Steffes M, Jacobs DR. Association between serum concentrations of persistent organic pollutants and insulin resistance among nondiabetic adults: results from the National and Nutrition Examination Survey 1999-2002. Diabetes Care. 2007;30(3):622-8.

30. Lee DH, Lee IK, Porta M, Steffes M, Jacobs DR. Relationship between serum concentrations of persistent organic pollutants and the prevalence of metabolic syndrome among non-diabetic adults: results from the National and Nutrition Examination Survey 19992002. Diabetologia. 2007;50(9):1841-51.

31. Ha MH, Lee DH, Jacobs DR. Association between serum concentrations of persistent organic pollutants and self-reported cardiovascular disease prevalence: results from the National Health and Nutrition Examination Survey, 1999-2002. Environ Health Perspect. 2007;115(8):1204-9.

32. Arsenescu V, Arsenescu RI, King V, Swanson H, Cassis LA. Polychlorinated biphenyl-77 induces adipocyte differentiation and proinflammatory adipokines and promotes obesity and atherosclerosis. Environ Health Perspect. 2008;116(6):761-8.

33. Song MO, Freedman JH. Activation of mitogen activated protein kinases by PCB126 $\left(3,3^{\prime}, 4,4^{\prime}, 5\right.$-pentachlorobiphenyl) in HepG2 cells. Toxicol Sci. 2005;84(2):308-18.

34. Hennig B, Hammock BD, Slim R, Toborek M, Saraswathi V, Robertson LW. PCB-induced oxidative stress in endothelial cells: modulation by nutrients. Int J Hyg Environ Health. 2002;205(1-2): 95-102.

35. Matsusue K, Ishii Y, Ariyoshi N, Oguri K. A highly toxic coplanar polychlorinated biphenyl compound suppresses $\Delta 5$ and $\Delta 6$ desaturase activities which play key roles in arachidonic acid synthesis in rat liver. Chem Res Toxicol. 1999;12(12):1158-65.

36. Mandal PK, Bandyopadhyay A. Ambient air quality in the Kolkata Municipal Corporation Area: assessment of practices and policy from 2003 to 2010 with policy recommendations for air quality improvement. Environ Qual Manag. 2013;23(1):83-106.

37. Benson HJ. Microbiological applications: laboratory manual in general microbiology (8th ed.). Boston. McGraw-Hill. 2001.

38. UGC. Awareness about ill-effects of fireworks and upkeep of clean environment, a notification dated 11.12.2015 issued by the University Grants Commission (UGC), India on 14.12.2015. Available at http://www.ugc.ac.in/pdfnews/8074542 UGC-letter14-12-2015.pdf accessed on 29.04.2016. 